\title{
Painel híbrido OSB/MDP de madeira Pinus taeda e resina poliuretana à base de óleo de mamona
}

\author{
OSB/ MDP hybrid wood-panel with Pinus taeda wood \\ species and castor-oil resin
}

\section{Fabiane Salles Ferro \\ Tiago Hendrigo de Almeida \\ Amós Magalhães de Souza \\ Diego Henrique de Almeida \\ André Luis Christoforo \\ Francisco Antonio Rocco Lahr}

\section{Resumo}

${ }^{1}$ Fabiane Salles Ferro ${ }^{1}$ Universidade Estadual do Centro

Riozinho - PR - Brasil

${ }^{2}$ Tiago Hendrigo de Almeida

2Universidade de São Paulo São Carlos - SP - Brasil

${ }^{3}$ Amós Magalhães de Souza ${ }^{3}$ Universidade Federal dos Vales do $J$ equitinhonha e Mucuri Janaúba - MG - Brasil

${ }^{4}$ Diego Henrique de Almeida ${ }^{4}$ Universidade Federal de Rondônia Porto Velho - RO - Brasil

${ }^{5}$ André Luis Christoforo ${ }^{5}$ Universidade Federal de São Carlos São Carlos - SP - Brasil

${ }^{6}$ Francisco Antonio Rocco Lahr ${ }^{6}$ Universidade de São Paulo São Carlos - SP - Brasil

Recebido em 24/10/17 Aceito em 27/08/18

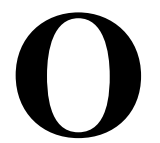

s painéis reconstituídos à base de madeira são uma alternativa para utilização na construção civil e na indústria do mobiliário. Dentre os tipos de painéis, destacam-se o OSB (Oriented Strand Board) e o MDP (Medium Density Particleboard). O objetivo desta pesquisa foi avaliar as propriedades físicas e mecânicas de painéis híbridos OSB/MDP, visando uma alternativa de uso em novo sistema fabril com o reaproveitamento de partículas de madeira. Foram fabricados painéis OSB, híbridos e MDP em escala laboratorial e determinadas suas respectivas propriedades físicas e mecânicas de acordo com as normas EN 300:2006 e ABNT NBR 14.810-2:2013. A influência dos tipos de painéis nas propriedades foi investigada pela análise de variância (ANOVA), os testes de normalidade de Anderson-Darling (AD) e de homogeneidade de variâncias de Bartlett (Bt) foram utilizados na verificação da validade das hipóteses da ANOVA. O teste de comparações múltiplas de Tukey foi utilizado para o agrupamento dos níveis do fator (tipo de painel) nas propriedades avaliadas. Os valores obtidos para as propriedades físicas e mecânicas dos painéis híbridos OSB/MDP alcançaram os valores mínimos determinados pelas normas vigentes. Os painéis híbridos apresentaram propriedades que permitem sua aplicação estrutural e em ambientes úmidos.

Palavras-chave: Painel de madeira. Resíduos de madeira. Propriedades físicas. Propriedades mecânicas.

\section{Abstract}

Reconstituted wood-based panels are an alternative for use in the building and furniture industries. Two important examples of them are the OSB (Oriented Strand Board) and the MDP (Medium Density Particleboard). The aim of this study was to evaluate the physical and mechanical properties of OSB/MDP hybrid panels, aiming at their use in a new wood particle recycling system. OSB, hybrids and MDP panels were manufactured at laboratory scale and their physical and mechanical properties were evaluated according to EN 300:2006 and ABNT NBR 14.810-2:2013 Codes. The influence of the panel types on physical and mechanical properties was investigated by analysis of variance (ANOVA), and Anderson-Darling $(A D)$ and Bartlett (Bt) variance homogeneity tests were used to verify the validity of the ANOVA. The Tukey multiple comparison test was used to group factor levels (panel type) in the evaluated properties. The values obtained for the physical and mechanical properties of the hybrid OSB/MDP panels reached the minimum values required by the current standards. The hybrid woodpanels presented properties that allow their application as structural members and their use in humid environments.

Keywords: Mechanical properties. Physical properties. Wood panel. Wood waste.

FERRO, F. S.; ALMEIDA, T. H. de; SOUZA, A. M. de; ALMEIDA, D. H. de; CHRISTOFORO, A. L.; LAHR, F. A. R. Painel híbrido OSB/MDP de madeira Pinus taeda e resina poliuretana à base de óleo de mamona. Ambiente Construído, Porto Alegre, v. 19, n. 3, p. 7-14, jul./ set. 2019. 


\section{Introdução}

A versatilidade da madeira permite sua utilização em vários seguimentos da economia (CHRISTOFORO et al., 2017; TAKESHITA; JANKOWSKY, 2015; MARINHO et al., 2017). Desde a sua extração na floresta nativa ou plantada (DOUGHERTY; WRIGHT, 2012; STEEGE et al., 2016), passando pelos processos industriais, consumidor e descarte final, a geração de resíduos acontece, e pode ser minimizada com o estudo prévio para o racional processamento da madeira (MANHIÇA; ROCHA; TIMOFEICZYK JUNIOR, 2013).

Estima-se que o aproveitamento da madeira em produtos finais, após seu processamento em serrarias, varie entre $40 \%$ e $60 \%$, sendo os resíduos gerados, na maioria das vezes pó de serra, descartados de maneira indiscriminada (VIEIRA et al., 2010; OFOEGBU; OGBONNAYA; BABALOLA, 2014; SILVA et al., 2017; MONTEIRO et al., 2017; ALMEIDA et al., 2017).

O Plano Nacional de Resíduos Sólidos (BRASIL, 2017) alia a geração de resíduos de madeira à necessidade do seu emprego e reciclagem na fabricação de painéis reconstituídos, tais como: o compensado, o MDP (Medium Density Particleboard), o MDF (Medium Density Fiberboard) e o OSB (Oriented Strand Board) (CHIROMITO et al., 2016; RUZIAK et al., 2017; NASCIMENTO et al., 2017; FERREIRA; SILVA; CAMPOS, 2017; SOUZA et al., 2018).

Os painéis OSB podem ser utilizados na fabricação de elementos estruturais para construção civil (vigas do tipo I-joints; lajes diafragmas; e fechamento de paredes estruturais autoportantes). Os painéis MDP são utilizados como matéria-prima para fabricação de móveis, entre outros artefatos da indústria do mobiliário (IWAKIRI et al., 2005, 2014; SANTOS et al., 2014; NASCIMENTO et al., 2015; ROSA et al., 2017).

Nesse sentido, destaca-se a fabricação de painéis híbridos à base de madeira. $\mathrm{O}$ painel híbrido recebe esse nome por contemplar partículas de madeira de diferentes dimensões (e espécies), com a possibilidade da inserção de outros tipos de materiais (fibras naturais ou sintéticas, por exemplo) em um mesmo painel (ALVES et al., 2014; CRAVO et al., 2015; BATTISTELLE et al., 2016; FERRO et al., 2016; VIDIL et al., 2016; ZHONG et al., 2017).

A fabricação de painéis híbridos pode ser uma alternativa que visa superar algumas limitações em termos de propriedades mecânicas dos painéis MDP e estabilidade dimensional dos painéis OSB, incrementando a gama de utilização desses produtos derivados da madeira, além de ser uma forma de aproveitamento de resíduos.

O objetivo desta pesquisa foi avaliar as propriedades físicas e mecânicas de painéis híbridos OSB/MDP, visando uma alternativa de uso em novo sistema fabril com reaproveitamento de partículas de madeira.

\section{Materiais e métodos}

Para o desenvolvimento deste estudo foram utilizadas partículas de madeira da espécie Pinus taeda, e resina poliuretana (PU) à base de óleo de mamona. A madeira foi adquirida em serrarias da região do município de São Carlos, interior de São Paulo, com $12 \%$ de teor de umidade no momento de fabricação das partículas. A resina PU-mamona era composta pelo poliol (derivado do óleo vegetal) e pelo isocianato polifuncional (pré-polímero), derivado do petróleo, os quais foram utilizados neste estudo na proporção de 1:1.

A utilização da resina PU-manona, assim como a proporção entre os seus componentes, justifica-se pelos excelentes resultados obtidos em estudos anteriores com painéis derivados de madeira, além do melhor desempenho ambiental quando comparadas com outras resinas utilizadas na fabricação de painéis de madeira (SILVA et al., 2013; LIANG; LV; YANG, 2016; ZHONG et al., 2017; SOUZA et al., 2018).

Para a geração das partículas strand, utilizadas na fabricação de OSB, peças de madeira foram seccionadas em dimensões de aproximadamente 90 mm de largura e $35 \mathrm{~mm}$ de espessura. $\mathrm{O}$ picador de disco foi regulado para que os strands possuíssem espessura da ordem de $0,7 \mathrm{~mm}$. As partículas homogêneas para fabricação dos painéis MDP foram obtidos a partir dos resíduos do processamento da madeira para fabricação dos strands. Os resíduos de madeira foram processados em um moinho-martelo com peneira de 2,8 mm.

Foram avaliadas três condições experimentais relacionadas a cada tipo de painel fabricado:

(a) condição 1 (C1): OSB;

(b) condição 2 (C2): Painel Híbrido; e

(c) condição 3 (C3): MDP.

Para cada condição experimental foram produzidos 4 painéis, com densidade de $0,75 \mathrm{~g} / \mathrm{cm}^{3}$, totalizando 12 painéis. Em todas as condições experimentais, as partículas foram encoladas com $12 \%$ de resina poliuretana à base de óleo de mamona (Figura 1a), em relação à massa seca de partículas. 
O OSB foi fabricado em três camadas, com espessura final de $10 \mathrm{~mm}$. Distribuídas manualmente, as partículas das camadas externas foram dispostas de forma orientada no sentido longitudinal do painel, enquanto as partículas da camada interna foram distribuídas de forma aleatória. A relação em massa de partículas das camadas externas e interna adotada foi de 20:60:20 (FERRO et al., 2015).

O painel híbrido foi produzido de acordo com o mesmo método empregado na fabricação do OSB. As camadas externas foram compostas de partículas strands, as quais foram orientadas no sentido longitudinal do painel (Figura 1b), enquanto as partículas homogêneas foram adicionadas ao colchão de partículas para compor a camada interna (Figura 1c). Com relação à fabricação dos painéis de partículas homogêneas, as partículas foram encoladas com a resina poliuretana à base de óleo de mamona e posteriormente adicionadas ao molde do painel.

Os colchões de partículas (Figura 1d) das três condições experimentais avaliadas foram préprensados para conformação das partículas, e em seguida prensados em uma prensa hidráulica (Figura 1e) com temperatura de $100{ }^{\circ} \mathrm{C}$ por 10 minutos e pressão específica de $4 \mathrm{MPa}$. Para estabilização e cura completa da resina, as chapas foram acondicionadas por 48 horas, em condição ambiente (Figura 1f). Após esse período elas foram esquadrejadas, para posterior retirada dos corpos de prova para os ensaios físicos e mecânicos. Na Figura 1 estão apresentadas as etapas de produção dos painéis híbridos.

A adoção de tais parâmetros para fabricação dos painéis bem como as condições de prensagem se justificam pelos excelentes resultados obtidos em estudos com painéis compósitos à base de madeira juntamente com resina poliuretana à base de óleo de mamona (BERTOLINI et al., 2014; FERRO et al., 2016; NASCIMENTO et al., 2017; SOUZA et al., 2018).

Foram realizados ensaios para determinação das propriedades mecânicas como módulo de elasticidade $(E)$ e resistência $\left(f_{\mathrm{M}}\right)$ à flexão estática e adesão interna (RTP), e para as propriedades físicas como inchamento em espessura após 2 horas (IE2h) e 24 horas (IE24h) em imersão em água, e também a absorção de águas após 2 horas (A2h) e 24 horas (A24h). Os ensaios mecânicos para determinação das propriedades de flexão estática foram realizados em uma máquina universal de ensaios EMIC com capacidade de $30 \mathrm{kN}$ e velocidade constante de 5 $\mathrm{mm} / \mathrm{min}$, enquanto o ensaio de adesão interna foi realizado em uma máquina universal de ensaios AMSLER com capacidade de $25 \mathrm{kN}$.

\section{Figura 1 - Processo de fabricação dos painéis híbridos}

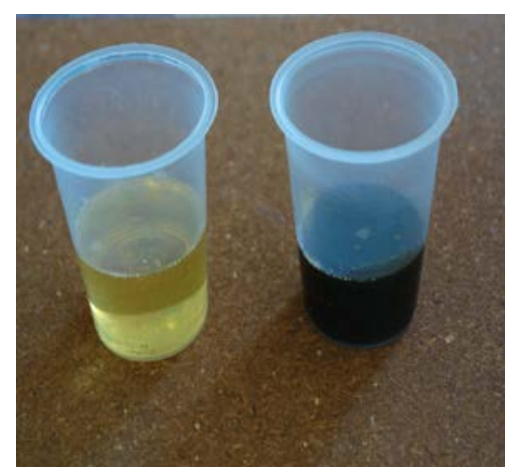

(a) Resina poliuretana bicomponente

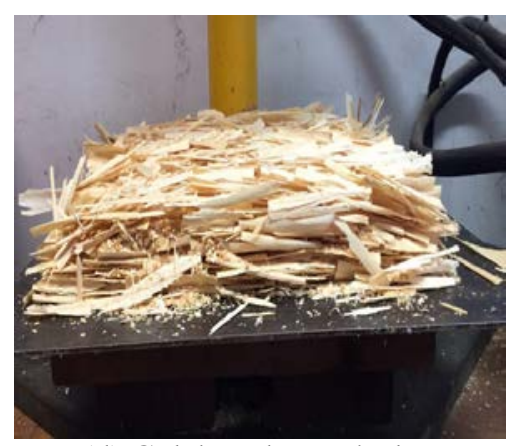

(d) Colchão de partículas

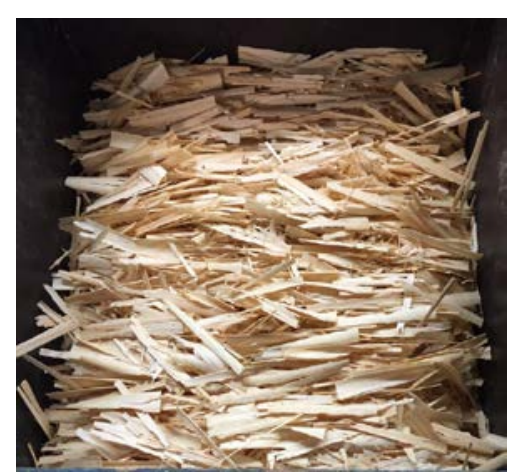

(b) Camada interna com partículas de MDP dos painéis híbridos

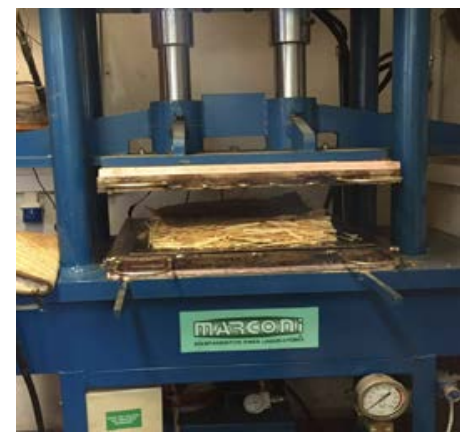

(e) Prensagem do colchão de partículas

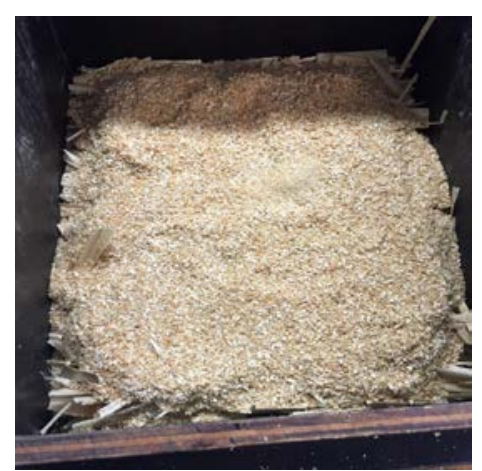

(c) Camada externa com partículas de OSB dos painéis híbridos

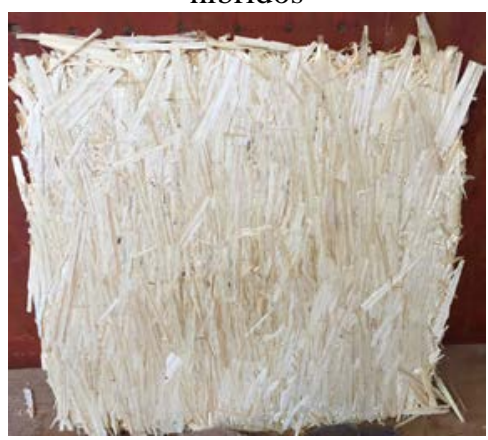

(f) Acondicionamento dos painéis híbridos 
Os ensaios do OSB e painel híbrido foram procedidos de acordo com a norma EN 300 (EUROPEAN..., 2006) “Oriented Strand Boards (OSB) - Definitions, classification and specifications". Os ensaios físicos e mecânicos supracitados também foram realizados para os painéis de partículas homogêneas; todavia, foram realizados com base na norma NBR 14810-2 (ABNT, 2013) "Painéis de partículas de média densidade” (ABNT, 2013).

A influência dos tipos de painéis (OSB, Híbrido, MDP) nas propriedades físicas e mecânicas foi investigada pela análise de variância (ANOVA), considerada ao nível de $5 \%$ de significância $(\alpha)$. O teste de comparações múltiplas de Tukey foi utilizado para o agrupamento dos níveis do fator (tipo de painel) nas propriedades avaliadas.

Os testes de normalidade de Anderson-Darling (AD) e de homogeneidade de variâncias de Bartlett (Bt) foram utilizados na verificação da validade das hipóteses do modelo da ANOVA. Pela formulação de ambos os testes, também considerados ao nível de 5\% de significância.

A Tabela 1 apresenta o número de determinações obtidas para cada tipo de painel e por propriedade avaliada, totalizando 333 resultados experimentais obtidos.

\section{Resultados e discussão}

A Tabela 2 apresenta os valores médios $(\overline{\mathrm{x}})$ e os coeficientes de variação (CV) obtidos para as propriedades físicas e mecânicas para os três tipos de painéis avaliados nesta pesquisa.

De acordo com a Tabela 3, para as propriedades físicas analisadas como: IE2h, IE24h, A2h e A24h, os menores valores foram obtidos para o MDP, seguido pelo painel híbrido e OSB.

As normas EN 300 (EUROPEAN..., 2006) e NBR 14810-2 (ABNT, 2013) fazem apenas referência à propriedade IE24h. Comparando-se os valores médios de IE24h obtidos neste estudo com os valores de referência da norma EN 300 (EUROPEAN..., 2006), foi possível classificar o
OSB e o painel híbrido, respectivamente, como painéis destinados a ambientes secos, cujo máximo valor de IE24h permitido é $25 \%$, e painéis destinados a utilizações em ambientes úmidos, nos quais o máximo valor de IE24h permitido é $20 \%$. De acordo com a norma NBR 14810-2 (ABNT, 2013), o MDP pode ser classificado como não estrutural destinados a ambientes úmidos, cujo máximo valor de IE24h permitido é $17 \%$.

Confrontando os valores de IE2h com os do estudo de Bertolini et al. (2014), notou-se que o MDP teve resultado coerente com o valor de $9,2 \%$ encontrado pelos autores para painéis de partículas homogêneas fabricados com Pinus sp. e resina poliuretana à base de óleo de mamona.

Com relação aos valores encontrados para as propriedades A2h e A24h, para painéis MDP fabricados com madeira de Eucalyptus urophylla e resina ureia formaldeído, Bianche et al. (2012) obtiveram valores médios de $17,4 \%$ e $57,6 \%$, respectivamente. Os maiores valores encontrados por esses autores podem ser justificados pela maior resistência à água da resina poliuretana à base de óleo de mamona utilizada neste estudo.

Verificou-se que para as propriedades mecânicas, E e $f_{\mathrm{M}}$, como esperado, os maiores valores foram obtidos pelo OSB, seguido pelo painel híbrido, e por último o MDP. Partículas mais compridas, como as utilizadas na fabricação do OSB e nas camadas externas do painel híbrido, contribuem para maiores valores de $\mathrm{E}$ e $f_{\mathrm{M}}$, fato este que pode ser atribuído aos maiores valores de propriedades mecânicas na direção paralela às fibras da madeira.

Comparando os resultados obtidos de $\mathrm{E}$ e $f_{\mathrm{M}}$ com os valores de referência estabelecidos pela norma EN 300 (EUROPEAN..., 2006) de $4.800 \mathrm{MPa}$ e 30 $\mathrm{MPa}$, respectivamente, foi possível classificar tanto o OSB quanto o painel híbrido como painéis estruturais com alta capacidade de resistência destinados a ambientes úmidos. Para o MDP, os resultados obtidos neste estudo foram coerentes com os recomendados pela norma NBR 14810-2 (ABNT, 2013) para painéis do tipo não estrutural, cujos valores recomendados são de $2.050 \mathrm{MPa}$ e 15 $\mathrm{MPa}$, para $\mathrm{E}$ e $f_{\mathrm{M}}$, respectivamente.

Tabela 1 - Número de determinações por painel e por propriedade avaliada

\begin{tabular}{c|c|c|c}
\hline Propriedades & Híbrido & OSB & MDP \\
\hline IE2h & 11 & 12 & 18 \\
IE24h & 11 & 12 & 18 \\
A2h & 11 & 12 & 18 \\
A24h & 11 & 12 & 18 \\
E & 29 & 12 & 20 \\
$f_{M}$ & 29 & 12 & 20 \\
RTP & 15 & 12 & 20 \\
\hline
\end{tabular}


Tabela 2 - Resultados obtidos na determinação das propriedades físicas e mecânicas dos painéis OSB, híbrido e MDP

\begin{tabular}{l|c|c|c|c|c|c}
\hline \multirow{2}{*}{ Propriedades } & \multicolumn{2}{|c|}{ OSB } & \multicolumn{2}{c|}{ Híbrido } & \multicolumn{2}{c}{ MDP } \\
\cline { 2 - 7 } & $\overline{\mathbf{x}}$ & $\mathbf{C V ( \% )}$ & $\overline{\mathbf{x}}$ & $\mathbf{C V ( \% )}$ & $\overline{\mathbf{x}}$ & $\mathbf{C V ( \% )}$ \\
\hline IE2h (\%) & 14,0 & 29,20 & 13,78 & 26,38 & 7,09 & 18,55 \\
IE24h (\%) & 25,2 & 14,74 & 18,82 & 18,90 & 11,00 & 9,61 \\
A2h (\%) & 34,0 & 34,01 & 16,18 & 27,21 & 14,90 & 45,22 \\
A24h (\%) & 34,4 & 29,51 & 28,65 & 19,90 & 52,80 & 20,30 \\
E (MPa) & 8.238 & 8,21 & 6.213 & 14,62 & 2.131 & 15 \\
$f_{\mathrm{M}}(\mathrm{MPa})$ & 54,8 & 8,40 & 53,32 & 18,35 & 20,60 & 15,52 \\
RTP (MPa) & 1,58 & 24,63 & 2,32 & 24,47 & 3,30 & 26,52 \\
\hline
\end{tabular}

Tabela 3 - Resultados da ANOVA, validação da ANOVA e do teste de Tukey

\begin{tabular}{c|c|c|c|c|c|c}
\hline \multirow{2}{*}{ Propriedades } & \multirow{2}{*}{ ANOVA } & \multicolumn{2}{|c|}{ Validação ANOVA } & \multicolumn{3}{c}{ Tukey } \\
\cline { 3 - 7 } & & AD & BT & OSB & Híbrido & MDP \\
\hline IE2h & 0,000 & 0,920 & 0,101 & $\mathrm{~A}$ & $\mathrm{~A}$ & $\mathrm{~B}$ \\
IE24h & 0,000 & 0,960 & 0,122 & $\mathrm{~A}$ & $\mathrm{~B}$ & $\mathrm{C}$ \\
$\mathrm{A} 2 \mathrm{~h}$ & 0,000 & 0,753 & 0,089 & $\mathrm{~A}$ & $\mathrm{~B}$ & $\mathrm{~B}$ \\
$\mathrm{~A} 24 \mathrm{~h}$ & 0,000 & 0,623 & 0,064 & $\mathrm{~A}$ & $\mathrm{~B}$ & $\mathrm{~B}$ \\
$\mathrm{E}$ & 0,000 & 0,178 & 0,102 & $\mathrm{~A}$ & $\mathrm{~B}$ & $\mathrm{C}$ \\
$\mathrm{fM}_{\mathrm{M}}$ & 0,000 & 0,136 & 0,098 & $\mathrm{~A}$ & $\mathrm{~A}$ & $\mathrm{~B}$ \\
$\mathrm{RTP}$ & 0,000 & 0,076 & 0,082 & $\mathrm{C}$ & $\mathrm{B}$ & $\mathrm{A}$ \\
\hline
\end{tabular}

Para os painéis OSB e híbrido, os resultados obtidos neste estudo para as propriedades $\mathrm{E}$ e $f_{\mathrm{M}}$ foram próximos aos determinados por Ferro et al. (2015) para painéis OSB fabricados com madeira de paricá (Schizolobium amazonicum) e resina PU-mamona.

Para os painéis MDP, os resultados de $\mathrm{E}$ e $f_{\mathrm{M}}$ foram coerentes com os de Bertolini et al. (2014) que encontraram valores médio de 2.109 $\mathrm{MPa}$ e 19 $\mathrm{MPa}$, respectivamente, para painéis de partículas homogêneas fabricados com madeira de Pinus sp. e resina PU-mamona.

Os valores de RTP obtidos pelo OSB e painel híbrido foram superiores ao valor mínimo de 0,5 MPa recomendados pela EN 300 (EUROPEAN..., 2006) para painéis estruturais com alta capacidade de carga destinados a ambientes úmidos. O mesmo ocorreu para os painéis MDP, cujo valor de RTP foi superior ao valor mínimo de 0,75 $\mathrm{MPa}$ recomendado pela norma NBR 14810-2 (ABNT, 2013) para painéis estruturais para uso em condições severas de carga, em ambientes úmidos. Os resultados de RTP do MDP apresentados nesta pesquisa são superiores aos apresentados por Iwakiri et al. (2014) para painéis aglomerados fabricados com Pinus taeda e Melia azedarach e 8\% de resina ureia-formaldeído, e obtiveram valores de RTP de 0,86 MPa e 1,55 MPa, respectivamente. A Tabela 3 apresenta os resultados obtidos na realização dos testes estatísticos.

Da Tabela 3, pelos P-valores dos testes de normalidade e homogeneidade de variâncias entre os tratamentos para todas as respostas investigadas serem superiores a $5 \%$, constata-se que as distribuições são normais e as variâncias são equivalentes, validando o modelo da ANOVA. Da ANOVA (Tabela 3), pode-se observar que a geometria dos strands afetou diretamente as propriedades físicas e mecânicas dos painéis investigadas neste estudo.

Para as propriedades físicas, a substituição dos strands por partículas homogêneas no interior do painel OSB (painel híbrido) proporcionou reduções significativas no inchamento em espessura após 24 h de imersão em água, quando comparado ao painel OSB convencional. Como mencionado, o OSB segundo a EN 300 (EUROPEAN..., 2006) foi classificado como painel estrutural destinado à utilização em ambientes secos, enquanto o painel híbrido foi classificado, segundo a referida norma, como painel estrutural destinado à utilização em ambientes úmidos. Confrontando as demais propriedades físicas investigadas do OSB com o painel híbrido (Tabela 3), foi possível observar que o IE2h, A2h e A24h também apresentaram reduções significativas, devido à substituição dos strands por partículas homogêneas.

Diferenças significativas também foram observadas para as propriedades mecânicas. Quando comparado ao OSB, o painel híbrido apresentou menores valores de E, $f_{\mathrm{M}}$ e RTP; todavia, os valores obtidos por esse último painel atendem aos máximos valores recomendados pela norma europeia para aplicação em estruturas especiais. 


\section{Conclusões}

Foi possível concluir, através dos resultados obtidos da presente pesquisa, que:

(a) os valores obtidos para as propriedades físicas do painel híbrido OSB/MDP alcançaram os valores mínimos recomendados pelas normas vigentes; e

(b) o painel híbrido apresentaram propriedades que permitem sua aplicação estrutural e em ambientes úmidos.

\section{Referências}

ALMEIDA, D. H. et al. Analysis of Solid Waste Generation in a Wood Processing Machine. International Journal of Agriculture and Forestry, v. 7, p. 76-79, 2017.

ALVES, L. S. et al. Particleboard Produced With Sawmill Waste of Different Wood Species.

Advanced Materials Research, v. 884-885, p. 689-693, 2014.

ASSOCIAÇÃO BRASILEIRA DE NORMAS TÉCNICAS. NBR 14.810-2: painéis de partículas de média densidade. Rio de Janeiro, 2013.

BATTISTELLE, R. A. G. et al. Physical and Mechanical Characterization of Sugarcane Bagasse Particleboards For Civil Construction. Journal of Sustainable Development of Energy, Water and Environmental Systems, v. 4, p. 408-417, 2016.

BERTOLINI, M. S. et al. Painéis de Partículas Provenientes de Rejeitos de Pinus sp. Tratado Com Preservante CCA e Resina Derivada de Biomassa. Revista Árvore, v. 38, p. 339-346, 2014.

BIANCHE, J. J. et al. Propriedades de Painéis Aglomerados Fabricados Com Partículas de Eucalipto (Eucalyptus urophylla), Paricá (Schizolobium amazonicum) e Vassoura (Sida spp.). Cerne, v. 18, p. 623-630, 2012.

BRASIL. Ministério do Meio Ambiente, Política Nacional de Resíduos Sólidos. Disponível em: <http://www.mma.gov.br/port/conama/legiabre.cf m?codlegi=636>. Acesso em: 15 jul. 2017.

CHIROMITO, E. M. S. et al. Propriedades Mecânicas de Painéis Produzidos Com Lascas de Madeira de Três Diferentes Comprimentos. Scientia Forestalis, v. 44, p. 175-180, 2016.

CHRISTOFORO, A. L. et al. Physical-Mechanical Characterization of the Anandenanthera colubrine Wood Specie. Engenharia Agrícola, v. 37, p. 376-384, 2017.
CRAVO, J. C. M. et al. Manufacture of Particleboard Based on Cement Bag and Castor Oil Polyurethane Resin. Construction and Building Materials, v. 87, p. 8-15, 2015.

DOUGHERTY, D.; WRIGHT, J. Silviculture and Economic Evaluation of Eucalypt Plantations in the Southern US. BioResources, v. 7, p. 19942001, 2012.

\section{EUROPEN COMMITTEE FOR}

STANDARDIZATION. EN 300: Oriented Strand Boards (OSB): definitions, classification and specification. Brussels, 2006.

FERREIRA, B. S.; SILVA, J. V. F.; CAMPOS, C. I. Static Bending Strength of Heat-Treated and Chromated Copper Arsenate-Treated Plywood. BioResources, v. 12,p. 6276-6282, 2017.

FERRO, F. S. et al. Physical Properties of OSB Panels Manufactured With CCA and CCB Treated Schizolobium amazonicum and Bonded With Castor Oil Based Polyurethane Resin.

International Journal of Materials Engineering, v. 6, p. 151-154, 2016.

FERRO, F. S. et al. Produção de Painéis de Partículas Orientadas (OSB) Com Schizolobium amazonicum e Resina Poliuretana à Base de Óleo de Mamona. Scientia Forestalis, v. 43, p. 313-320, 2015.

IWAKIRI, S. et al. Avaliação do Potencial de Uso da Madeira de Acrocarpus fraxinifolius, Grevilea robusta, Melia azedarach e Toona ciliata Para Produção de Painéis OSB. Cerne, v. 20, p. 277284, 2014.

IWAKIRI, S. et al. Produção de Painéis de Madeira Aglomerada de Alta Densificação Com Diferentes Tipos de Resinas. Scientia Forestalis, v. 68, p. 39-43, 2005.

LIANG, W.; LV, M.; YANG, X. The Combined Effects of Temperature and Humidity on Initial Emittable Formaldehyde Concentration of a Medium-Density Fiberboard. Building and Environment, v. 98, p. 80-88, 2016.

MANHIÇA, A; A.; ROCHA, M. P.; TIMOFEICZYK JUNIOR, R. Custos do Desdobro de Pinus spp. Com Utilização de Modelos de Corte Numa Serraria. Floresta e Ambiente, v. 20, p. 327-335, 2013.

MARINHO, N. P. et al. Características da Polpa kraft Extraída da Espécie Acácia-Negra na Produção de Papel. Floresta e Ambiente, v. 24, p. e00099214, 2017.

MONTEIRO, T. C. et al. Energy Balance in Sawing Eucalyptus grandis Logs. BioResources, v. 12, p. 5.790-5.800, 2017. 
NASCIMENTO, M. F. et al. Painéis OSB Fabricados Com Madeiras da Caatinga do Nordeste do Brasil. Ambiente Construído, Porto Alegre, v. 15, n. 1, p. 41-48, jan./mar. 2015.

NASCIMENTO, M. F. et al. Roughness Study on Homogeneous Layer Panels Manufactured From Treated Wood Waste. Acta Scientiarium

Technology, v. 39, p. 27-32, 2017.

OFOEGBU, C.; OGBONNAYA, S.;

BABALOLA, F. D. Sawmill Conversion

Efficiency and Wood Recovery of Timber Species in Cross River State Nigeria. Agriculture and Forestry, v. 60, p. 105-113, 2014.

ROSA, T. S. et al. Utilização de Cinco Espécies de Eucalyptus Para a Produção de Painéis OSB.

Floresta e Ambiente, v. 24, p. e20160049, 2017.

RUZIAK, I. et al. Influence of Urea-Formaldehyde Adhesive Modification With Beech Bark on Chosen Properties of Plywood. BioResources, v. 12, p. 3250-3264, 2017.

SANTOS, W. L. F. et al. Particleboard Manufactured From Tauari (Couratari oblongifolia) Wood Waste Using Castor oil Based Polyurethane Resin. Materials Research, v. 17, p. 657-663, 2014.

SILVA, C. P. et al. Quantificação de Resíduos Produzidos nas Indústrias Madeireiras de Gurupi, TO. Floresta e Ambiente, v. 24, p. e00065613, 2017.
SILVA, S. A. M. et al. Painéis de Partículas de Madeira Leucena e resina Poliuretana Derivada de Óleo de Mamona. Ciência Rural, v. 43, p. 13991404, 2013.

SOUZA, A. M. et al. Wood-Based Composite Made of Wood Waste and Epoxy Based Ink-Waste Adhesive: a cleaner production alternative.

Journal of Cleaner Production, v. 193, p. 549562, 2018.

STEEGE, H. et al. The Discovery of the Amazonian Tree Flora With an Update Checklist of All Known Tree Taxa. Scientific Reports, v. 6, p. 1-15, 2016.

TAKESHITA, S.; JANKOWSKY, I. P. Redução na Movimentação Dimensional da Madeira de Jatobá (Hymenaea sp.) e Muiracatiara (Astronium sp.) Submetidas a Tratamento Térmico Adicional. Scientia Forestalis, v. 43, p. 345-352, 2015.

VIDIL, L. et al. Thermal Insulating Particle Boards Reinforced With Coconut Leaf Sheaths. Green Materials, v. 4, p. 31-40, 2016.

VIEIRA, R. S. et al. Small Wooden Objects Using Eucalypts Sawmill Wood Waste. BioResources, v. 5, p. 1.463-1.472, 2010.

ZHONG, R. et al. Impacts of Urea-Formaldehyde Resin Residue on Recycling and Reconstitution of Wood-Based Panels. International Journal of Adhesion and Adhesives, v. 78, p. 60-66, 2017. 


\section{Fabiane Salles Ferro}

Departamento de Engenharia Florestal | Universidade Estadual do Centro Oeste | PR 153, km 7 | Riozinho - PR - Brasil | CEP 84500-000 | Tel.: (16) 98204-4972 | E-mail: fabi.salles.ferro@gmail.com

\section{Tiago Hendrigo de Almeida}

Escola de Engenharia de São Carlos | Universidade de São Paulo | Av. Trabalhador Sancarlense, 400, Parque Arnold Schimidt | São Carlos - SP - Brasil | CEP 13566-590 | Tel.: (15) 99606-7897 | E-mail: tiago.hendrigo@gmail.com

\section{Amós Magalhães de Souza}

Departamento de Engenharia de Materiais | Universidade Federal dos Vales do J equitinhonha e Mucuri| Avenida Um, 4050, Cidade Universitária | J anaúba - MG - Brasil | CEP 39447-814 | E-mail: amosmag@gmail.com

\section{Diego Henrique de Almeida}

Departamento de Engenharia Civil, Universidade Federal de Rondônia | BR 364, km | Porto Velho - RO - Brasil | CEP $76801-059$ | Tel.: (69) 2182-2100 | E-mail: diegoestruturas@gmail.com

\section{André Luis Christoforo}

Departamento de Engenharia Civil | Universidade Federal de São Carlos | Rodovia Washington Luís, km 235 - SP-310 | São Carlos - SP Brasil | CEP 36307-352 | Tel.: (16) 3351-8262 | E-mail: christoforoal@yahoo.com.br

\section{Francisco Antonio Rocco Lahr}

Escola de Engenharia de São Carlos | Universidade de São Paulo | Tel.: (016) 3373-8206 | E-mail: frocco@sc.usp.br

\section{Revista Ambiente Construído}

Associação Nacional de Tecnologia do Ambiente Construído

Av. Osvaldo Aranha, 99 - 3o andar, Centro

Porto Alegre - RS - Brasil

CEP $90035-190$

Telefone: +55 (51) 3308-4084

Fax: +55 (51) 3308-4054

www. seer. ufrgs. br/ ambienteconstruido

E-mail: ambienteconstruido@ufrgs.br 